\title{
Study on Bridge Structure Damage and Health Diagnosis Method Based on Health Monitoring
}

\author{
Chunguang HAN, Xu LUO*, Xinping LI, Xinsha FU, Lixiong GU, Zhiyong OUYANG
}

\begin{abstract}
Due to the high cost of the bridge health monitoring system, only some large bridges are running the health monitoring system. This paper proposes methods for bridge structure damage and health diagnosis based on health monitoring. The establishment of various environmental load models, computer technology, and stochastic process analysis technology are equivalent to a large and complex system. The maximum longitudinal damage at the end fulcrum is about 24 mm, and the longitudinal damage does not exceed $6 \mathrm{kN}$. Moreover, the damage of the welding nail is caused by the temperature rise of $15^{\circ} \mathrm{C}$. The health monitoring of the bridge structure is ultimately to evaluate the health of the bridge structure. This paper analyses and evaluates the level of the cumulative damage of the bridge structure, which predicts the development trend of the damage, provides the bridge management department with timely status information, and provides bridge structure maintenance and management.
\end{abstract}

Keywords: bridge health monitoring; bridge structure; health diagnosis; structure damage

\section{INTRODUCTION}

With the rapid development of theory and technology in the field of design, construction technology and materials of bridge engineering, the development of cablestayed bridges is also attracting attention worldwide [1]. Thousands of long-span cable-stayed bridges have also been born. The rapid development of long-span bridges and the different severity of damage accidents in recent decades have aroused the attention of managers and everyone in the bridge engineering community on safety, durability, and normal use functions during the operation of cable-stayed bridges [2]. The vigorous development of technologies and artificial intelligence has given new vitality to bridge health monitoring, which is moving towards intelligence. In order to ensure the safety of the bridge structure during the construction phase, the performance of the bridge structure is evaluated in realtime monitoring data during the entire life cycle of the bridge, and the daily maintenance and maintenance of the bridge during the construction phase and service phase are smoothly performed.

The traditional health assessment of bridge structures is carried out by manual visual inspection of information obtained by means of portable instruments. Because these traditional inspection methods require many human, material, and financial resources, they are subjective, difficult to quantify, affect normal traffic, have long periods, and have poor real-time shortcomings and limitations. Based on this, the health monitoring system of modern long-span bridge structure is a modern advanced monitoring technology, data analysis and processing technology, health diagnosis technology and traditional manual inspection methods.

This paper mainly describes the development of large bridge health monitoring systems, the concept and composition of health monitoring systems, the design of health monitoring systems, and the theoretical development of health monitoring systems. The structure of this article is as follows. The introduction introduces the research background and current status of bridge structural damage and health diagnosis for health monitoring, and proposes the main thrust of this article. The second part presents the architecture and components of bridge structural health monitoring system. The design and monitoring content of bridge structural health monitoring system is described. Part 4 shows the experimental verification and analysis. Finally, the last part summarizes the full text and the innovation.

\section{BRIDGE STRUCTURE HEALTH MONITORING SYSTEM}

The main function and purpose of the bridge structure's health monitoring system is to enable the load-bearing capacity, operation status, safety, and durability of the bridge structure during construction and operation to grasp online in real time $[4,5]$. The measurement-based model refers to a model established by performing statistical analysis on the data measured by the health detection system with statistical analysis tools. They are generally applied to real-time structural health monitoring work [6].

The bridge health monitoring system can realize realtime control data collection and processing to determine the location of structural damage and predict the actual bearing capacity of the structure. The realization of these functions depends on the cooperation of various components of the health monitoring system [7]. The bridge intelligent health monitoring system has the following three components.

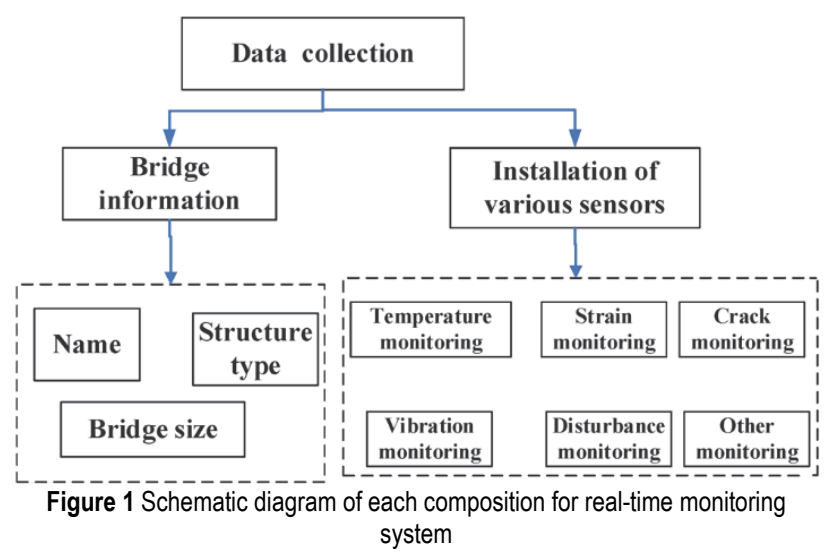

(1) Sensing subsystem

As shown in Fig. 1, this subsystem serves as the basic 
part of the system and is composed of various sensors required by the health monitoring content. The sensor resources are limited and are the main research directions of this subsystem.

(2) Data transmission subsystem

This subsystem is mainly composed of two parts: the conversion between the electrical signal of the sensor and the remote transmission of the signal. The conversion process among electrical signals needs to be denoised. The remote transmission of signals is divided into wired and wireless transmission. Compared with wired transmission, wireless transmission of data information changed from wire transmission to electromagnetic wave transmission, thereby reducing considerations such as preliminary wiring planning and site layout. The current research direction of wireless transmission is to reduce the data packet loss rate during the transmission process, which improves the data transmission efficiency [8]. The working principle of the subsystem: the sensor sends the collected data to the platform to achieve network storage and data release. Each user can obtain monitoring data at any time by accessing the platform through mobile phones or computer clients.

\section{(3) Real-time processing}

The damage identification and condition assessment subsystem is the most important subsystem of the entire bridge health monitoring system.

From the perspective of informatics, the process of bridge health monitoring is the process of extracting relevant information from data signals through testing to recognize the structure. The data processing and control system includes pre-processing, secondary processing, post-processing, data storage, data display, and data sharing. Data pre-processing is mainly to perform statistical calculations on the original data, calculate the maximum, minimum, mean, variance, standard deviation, and amplitude of change within a set period [9]. The calculation results are used as the input of the preliminary warning, which is used to determine whether the signal is normal. The secondary processing of data refers to the analysis of the data in the amplitude domain, display in the frequency domain, display in the time domain, display in the frequency count analysis, Fourier analysis, etc., in order to determine the development trend and characteristic value of the structural monitoring data for evaluation Basis for structural performance. Data post-processing refers to advanced analysis of monitoring data, such as: real-time modal analysis, correlation analysis between bridge feature quantities and environmental factors. Because it takes a long time to calculate, this analysis process needs to be performed offline.

\section{DESIGN AND MONITORING CONTENT OF BRIDGE STRUCTURAL HEALTH MONITORING SYSTEM 3.1 Monitoring Contents of Bridge Structural Health Monitoring System}

The health monitoring content of bridge structures usually depends on the purpose of monitoring and the type of bridge structure. Generally speaking, the monitoring content of bridge structures should include as follows: (1) Monitoring of the environment where the bridge structure is located, such as: atmospheric temperature, wind direction and wind speed, corrosion, pier foundation scouring conditions, ground pulsation, etc. Monitoring of environmental factors can determine the environmental load on the bridge structure. (2) Monitoring of the operational load of the bridge structure, such as road vehicles and train vehicles. The monitoring of operational loads can determine whether the bridge structure is overloaded during operation, and can establish a clear concept of the operational load model [13]. (3) Monitoring of structural characteristics, such as: static coefficient of influence of structure, dynamic characteristics of structure, and so on. (4) Monitoring the static and dynamic response of the structure, such as: geometric deformation of the overall structure of the structure, stress changes and distribution of key components and parts, fatigue status of steel components, cable force of stay cables, and so on.

Based on the reasonable processing and statistical analysis of the measured data of environmental load, operational load, and structural static and dynamic response, the statistical relationship model between environmental load and structural whole or local, operational load and structural whole or local can be established and obtained. It is also called measurementbased model. These models are the basis for real-time online health monitoring of long-span bridges [10]. The measurement data for these monitoring contents is also the basis for offline health assessment and injury identification based on a physics-based model.

\subsection{Design of Bridge Structure Health Monitoring System}

The design of the bridge structure health monitoring system should first follow the principles of "simple, practical, reliable performance, and economical reasonableness", while taking into account the requirements of bridge maintenance management and operation verification and scientific research needs. The data collection and transmission system should be automatic and continuous. The special collection and manual intervention can be performed under special circumstances. The system has a real-time self-diagnosis function and time synchronization function [11]. The data processing and control system first have reasonable and efficient pre-processing and secondary processing functions, laying a good foundation for offline postprocessing. Closely combine the management and maintenance requirements of each bridge, design a reasonable bridge health assessment, and damage identification system to diagnose the overall health of the bridge and the abnormal performance of the components, and find out the root cause, and find the catastrophic early Hidden danger. Design a reasonable inspection and maintenance system. The layout of a long-span cablestayed bridge health monitoring system in Hong Kong is shown in Fig. 2.

For the steel-concrete composite girder cable-stayed bridge, the overall and local static and dynamic responses under the effects of ambient temperature loads, wind loads, and vehicle loads during operation are the main content of setting up a health monitoring system. Specifically, the longitudinal displacements of the longitudinal bridges at both ends of the bridge system, the vertical displacements of the two midspans, the three-dimensional displacement of the tower top, the concentrated anchoring zone of the 
cable stays at the tower top, and the anchoring zone of the stay cables on the bridge. The stress and distribution of steel beams with mid-span cross-sections, and the vibration of the stay cables are all the important points to be monitored. Therefore, it can be seen from Fig. 2 that 83 temperature sensors for measuring atmospheric temperature and component temperature are respectively provided on the right midspan section and the two sections on the middle tower at different elevations. In order to realize wind monitoring, one anemometer on each of the three towers and a pair of seven anemometers on each of the two mid-span sections were used to measure the wind speed and direction [12].

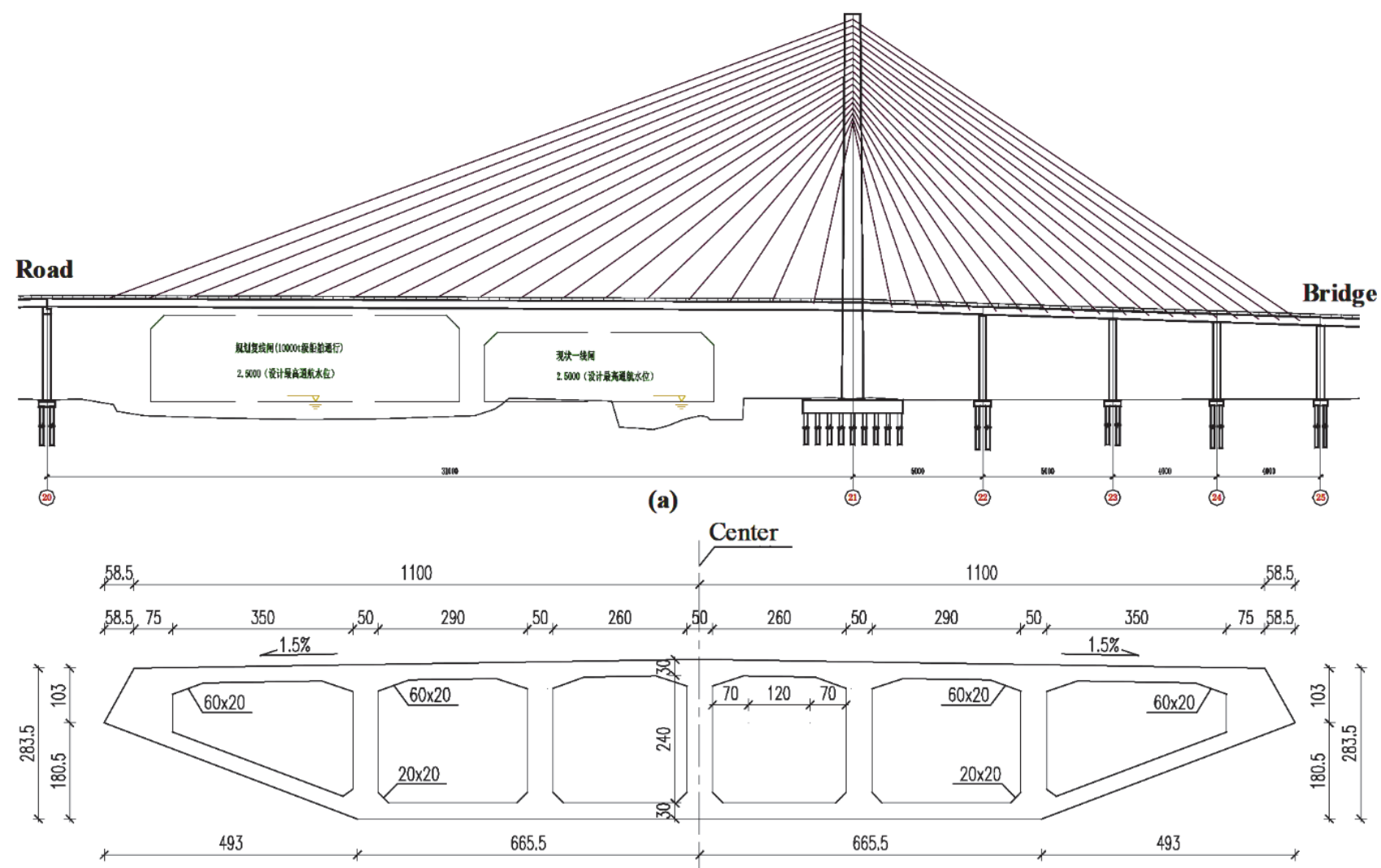

(b)

Figure 2 The structure of Bridge Structure Health Monitoring: (a) Bridge elevation; (b) Girder cross section

The data processing and analysis system of the health monitoring system and the health assessment system are set up in the bridge management center building attached to the bridge [17]. The data collected by the system includes the raw data obtained by each sensor and the preprocessed derived data. During the secondary and postprocessing of the data, it can be determined whether the data analysis should be based on the original data or whether to start with pre-processed data. The secondary data processing advanced data processing and structural health assessment of the health monitoring system all work offline.

\section{EXPERIMENTAL VERIFICATION AND ANALYSIS 4.1 Health Monitoring System Scheme Design}

In order to achieve the above monitoring purposes, it is necessary to analyze the data in conjunction with the changes in the environment. The sensor subsystem is a module composed of sensors that monitor parameters such as environmental parameters, stress, structural temperature, main beam deflection, main tower displacement, and structural dynamic characteristics [13]. Various sensors complete the direct collection task of monitoring parameters, and form different light or electrical signals for analysis and processing by the data acquisition module according to the different monitoring parameters. The sensor subsystem is divided into 6 sub-modules according to different monitoring objects, as shown in Tab. 1.

Table 1 Division of sensor subsystem

\begin{tabular}{|c|c|c|}
\hline \multicolumn{2}{|c|}{ Submodule } & Monitoring object \\
\hline 1 & $\begin{array}{c}\text { Temperature, humidity, wind } \\
\text { speed, etc. }\end{array}$ & $\begin{array}{c}\text { Temperature, humidity, wind } \\
\text { speed, etc. }\end{array}$ \\
\hline 2 & Deck traffic conditions & Deck traffic conditions \\
\hline 3 & $\begin{array}{c}\text { Bridge tower displacement, } \\
\text { main beam alignment }\end{array}$ & $\begin{array}{c}\text { Bridge tower displacement, main } \\
\text { beam alignment }\end{array}$ \\
\hline 4 & $\begin{array}{c}\text { Critical section strain (force }> \\
\text { and its changes }\end{array}$ & $\begin{array}{c}\text { Critical section strain (force }>\text { and } \\
\text { its changes }\end{array}$ \\
\hline 5 & Cable force and its change & Cable force and its change \\
\hline 6 & Girder and tower vibration & Girder and tower vibration \\
\hline
\end{tabular}

Because the environment of the bridge location is quite different from that of the meteorological station, and the bridge site is located near the sea, which is affected by the marine climate, environmental monitoring equipment should be installed at the bridge location. The main purpose and role of environmental monitoring is to select appropriate monitoring timing through environmental monitoring.

The whole bridge is equipped with 9 temperature and humidity meters, 2 anemometers and 2 trapezoidal anode 
corrosion monitors. Anemometers are installed on the top of the tower and in the main span to monitor the environmental wind speed, wind direction, and wind angle of attack. Thermo hygrometers are installed in the main tower and box girder, respectively, to monitor the temperature and relative humidity in the bridge tower and steel box girder. The position of the water level change and the trapezoidal anode corrosion monitor embedded in the main beam monitor the influence of the external environment on the durability of the structure.

The purpose of installing a video monitoring system on the bridge is to make the structural response monitoring subsystem work in conjunction with the video monitoring system. When the structural response subsystem reflects the stress indicators exceeding the limit, the bridge deck load can be obtained through the video monitoring system. Comparative analysis can also be used to monitor the driving conditions of overloaded vehicles on the bridge and make records to provide evidence for bridge monitoring and evaluation. At the same time, it is easy to trace the cause of traffic accidents above the bridge deck. In order to analyze the stress state and distribution of the full bridge, a spatial finite element program was used to simulate the transverse pre-stress of steel beams, concrete bridge decks, bottom slab concrete, steel beam longitudinal reinforcement system, steel beam diaphragm system, and concrete bridge decks.

The steel beam part adopts 4-node plate and shell elements, the concrete bridge deck, and bottom concrete adopts 8-node solid elements, the box beam horizontal braces and horizontal braces use space beam elements, and the transverse pre-stress of the concrete bridge deck is simulated by space rod elements. The bridge is divided into about 280,000 nodes and 330,000 units, and the unit size is about 0.3 to 0.5 meters. The model uses a Cartesian coordinate system, and the origin of the coordinates is located at the center of the bottom plate of the main beam at an end of 85 meters. The $X$-axis is the forward bridge direction, the $Y$-axis is the horizontal bridge direction, and the $Z$-axis is the vertical direction. Fig. 3 is the model diagram of the full bridge unit.

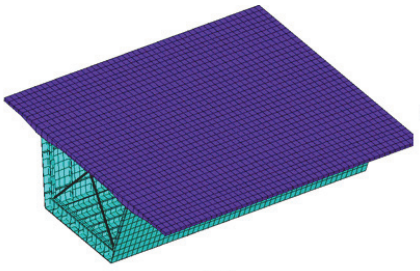

(a)

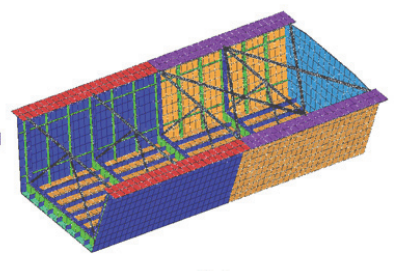

(b)
Figure 3 Main beam section beam model: (a) Main beam; (b) Bottom beam

\subsection{Data Acquisition System}

The data collection system needs to adopt two methods of threshold and timing. In the initial stage of the bridge structure operation, a 24-hour continuous acquisition strategy is adopted. After 30 days of operation, the data were analyzed to reveal the actual force characteristics and laws of the bridge structure. According to the actual force characteristics and laws of the bridge structure, the threshold determined to trigger the acquisition system, which determined the specific period for timing acquisition.
The design of the data acquisition system considers the overall structure of the data acquisition system, the software, hardware, and data acquisition strategies of the data acquisition system. The bridge is not very long and the signal attenuation is not obvious. Therefore, a data acquisition station is used for centralized data collection. The main beam bridge surface of the data acquisition station's tower-beam joint is shown in Fig. 4. To ensure the normal operation of the monitoring instrument, power must be supplied for 24 hours.

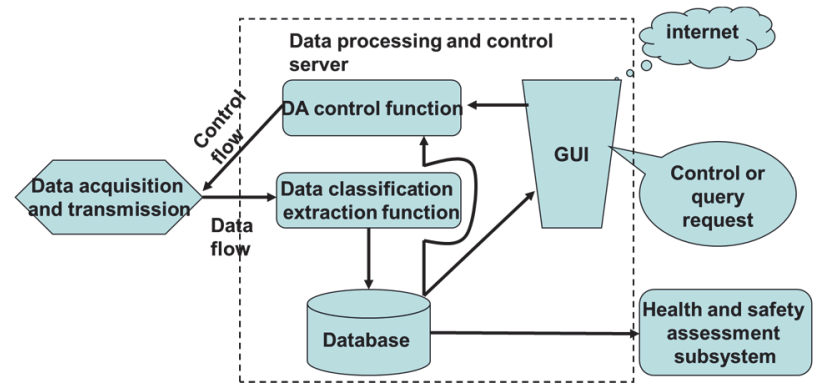

Figure 4 Composition of data processing and control subsystem

Structural deformation is the most sensitive and accurate reflection of structural state changes. Therefore, the monitoring of structural deformation can more accurately grasp the change of the internal load state of the structure at a constant load. In addition, some structural damage will also cause abnormal deformation conditions. Deformation monitoring can also identify these damages; the deformation of the bridge deck is directly related to the bridge alignment, and the applicability of the bridge can be judged by the change of the bridge alignment. Therefore, the monitoring of structural deformation is significant for internal force status and damage identification. Deformation monitoring can achieve the following purposes, which modify the finite element model for calculating the internal force of the structure, and judge the applicability of the bridge based on the monitoring results.

\subsection{Structural Damage Identification}

Structural damage identification is based on a certain mathematical model to predict the dynamic response parameters of the structure from experimentally measured data. Specific to the finite element model, that is, according to the structure identification theory, the mass matrix, stiffness matrix and damping matrix in the finite element model are inverted from the measured structural response data. The core of the existing structure recognition theory mainly includes the least square method theory and the maximum likelihood theory. The commonly used methods include extended filtering method, maximum likelihood method, recursive least square method, and action variable method. Ghanem and Shinozuka have reviewed these methods in detail, and compared these methods based on specific structural models. The comparison results show that the results sometimes given according to different theories are very different, which shows that the rationality and reliability of the existing test data need to be further improved.

According to the selected recognition accuracy, the structure recognition theory can identify the mass, stiffness and other parameters of each component in the structure. 
However, because the mass matrix, stiffness matrix, and damping matrix in the finite element model must be directly inverted, this method cannot be successfully used in large civil structures. (1) The stiffness matrix is mainly affected by the higher-order modes of the structure. Due to the limitation of the bandwidth of the test instrument, the measured modes are mainly low-order modes. (2) The parameters in the finite element model are mainly determined by the geometric shape and material properties of the structure, and have specific physical meanings. The "leakage frequency" phenomenon easily occurs during actual measurement, which makes the identified parameters deviate from their original physical meaning.

Due to different objective functions, the specific solution process is also very different, such as error matrix method, Bayesian estimation, etc. In many methods in the penalty function method, a sensitive matrix must be calculated. To calculate the sensitive matrix, the eigenvalues and eigenvectors must be calculated. The derivative of the parameter to modify is often very complicated. Only a few methods in the Lagrange multiplier method are reviewed below.

(1) Reference benchmark method

In the reference method, one of the mass matrix $\boldsymbol{M}$, the stiffness matrix $\boldsymbol{K}$, and the structural mode $\varphi$ must be known. The remaining two quantities are corrected through different minimization processes of the objective function. Berman and Nagy give the following correction process as follows.

$$
\boldsymbol{J}_{M}=\left\|\boldsymbol{M}_{\mathrm{A}}^{-\frac{1}{2}}\left(\boldsymbol{M}-\boldsymbol{M}_{\mathrm{A}}\right) \boldsymbol{M}_{\mathrm{A}}^{-\frac{1}{2}}\right\|+\sum_{i}^{m} \sum_{j}^{m} \lambda_{i j}\left(\varphi^{\mathrm{T}} \boldsymbol{M}^{\varphi}-\boldsymbol{I}\right)_{i j}
$$

$\lambda_{i j}$ is the Lagrange multiplier. The last term of Eq. (1) is to ensure the orthogonality of the eigenvectors during the mass matrix correction process. Among them, $\boldsymbol{M}_{\mathrm{A}}$ is the analysis model mass matrix. The stiffness matrix can be obtained by minimizing the following objective function:

$$
\begin{aligned}
& \boldsymbol{J}_{K}=\left\|\boldsymbol{M}_{\mathrm{A}}^{-\frac{1}{2}}\left(\boldsymbol{K}-\boldsymbol{K}_{\mathrm{A}}\right) \boldsymbol{M}_{\mathrm{A}}^{-\frac{1}{2}}\right\|+\sum_{i}^{p} \sum_{j}^{m} \lambda_{K i j} \times\left(\boldsymbol{K}^{\varphi}-\boldsymbol{M}^{\varphi} \boldsymbol{\Lambda}\right)_{i j}+ \\
& +\sum_{i}^{m} \sum_{j}^{m} \lambda_{O i j} \times\left(\varphi^{\mathrm{T}} \boldsymbol{K}^{\varphi}-\boldsymbol{\Lambda}\right)_{i j}+\sum_{i}^{p} \sum_{j}^{i} \lambda_{S i j}\left(\boldsymbol{K}-\boldsymbol{K}^{\mathrm{T}}\right)_{i j}
\end{aligned}
$$

In the formula, $\boldsymbol{\Lambda}$ is the spectral matrix. The Lagrange multiplier $\lambda S_{i j}$ is introduced to ensure the symmetry of the stiffness matrix, and the Lagrange multiplier $\lambda O_{i j}$ is introduced to ensure the orthogonality of the feature vector. The revised stiffness matrix is as follows.

$$
\boldsymbol{K}=\boldsymbol{K}_{\mathrm{A}}+\left(\boldsymbol{\Lambda}+\boldsymbol{\Lambda}^{\mathrm{T}}\right)
$$

In the above correction process, false modes will inevitably be generated, and the positive definiteness of the mass matrix and the stiffness matrix will be destroyed at the same time. The modified model cannot be used to calculate the dynamic response of the structure to obtain the dynamic stress in the structure, so it cannot be used for the fatigue damage analysis of the structure.

(2) Mixed matrix method
The main idea of the mixed matrix method is to jointly generate the mass matrix and stiffness matrix of the structure through the data of test and analysis. Since the order $m$ of the tested modal data is smaller than the order $p$ required for structural analysis, the high-order modal data that has not been tested will be replaced with the data analyzed by the original model. The modified mass matrix and stiffness matrix are as follows.

$$
\begin{aligned}
\boldsymbol{M}^{-1} & =\sum_{i=1}^{m} \varphi_{\mathrm{T} i} \varphi_{\mathrm{T} i}^{\mathrm{T}}+\sum_{i=m+1}^{p} \varphi_{\mathrm{T} i} \varphi_{\mathrm{T} i}^{\mathrm{T}} \\
\boldsymbol{K}^{-1} & =\frac{\sum_{i=1}^{m} \varphi_{\mathrm{T} i} \varphi_{\mathrm{T} i}^{\mathrm{T}}}{\omega_{\mathrm{T} i}^{2}}+\frac{\sum_{i=m+1}^{p} \varphi_{\mathrm{T} i} \varphi_{\mathrm{T} i}^{\mathrm{T}}}{\omega_{\mathrm{A} i}^{2}}
\end{aligned}
$$

In the formula, the subscripts $\mathrm{A}$ and $\mathrm{T}$ represent analysis and test data, respectively.

(3) Feature structure-matching method

The feature structure matching method mainly introduces the state feedback technology into the correction process. The correction assumes that the structure's mass matrix is known (i.e., no correction is required). After correction, the stiffness matrix and damping matrix of the structure can be obtained as follows.

$$
\begin{aligned}
& \boldsymbol{K}=\boldsymbol{K}_{\mathrm{A}}+\boldsymbol{B}_{0} \boldsymbol{G} \boldsymbol{C}_{0} \\
& \boldsymbol{C}=\boldsymbol{C}_{\mathrm{A}}+\boldsymbol{B}_{0} \boldsymbol{G} \boldsymbol{C}_{1}
\end{aligned}
$$

$\boldsymbol{B}_{0}$ is the input distribution matrix; $\boldsymbol{C}_{0}$ and $\boldsymbol{C}_{1}$ are matrices related to the output and state; $\boldsymbol{G}$ is the feedback gain matrix. $\boldsymbol{C}_{1}^{\varphi \Lambda}+\boldsymbol{C}_{0}^{\varphi}$ can be arbitrarily selected. The choice of $\boldsymbol{C}_{0}$ and $\boldsymbol{C}_{1}$ must make $\boldsymbol{C}_{1}^{\varphi \Lambda}+\boldsymbol{C}_{0}^{\varphi}$ non-singular. In general, the matrix $\boldsymbol{B}_{0} \boldsymbol{G} \boldsymbol{C}_{0}$ and matrix $\boldsymbol{B}_{0} \boldsymbol{G} \boldsymbol{C}_{1}$ are asymmetric. By constantly adjusting and making matrix $\boldsymbol{B}_{0} \boldsymbol{G} \boldsymbol{C}_{0}$ and matrix $\boldsymbol{B}_{0} \boldsymbol{G} \boldsymbol{C}_{1}$ a symmetric matrix, $\boldsymbol{K}$ and $\boldsymbol{C}$ are symmetric. The process of debugging $\boldsymbol{C}_{0}$ and $\boldsymbol{C}_{1}$ is very complicated. For large structures, the amount of calculation will be huge. Therefore, this method is also difficult to be used to modify the large-scale bridge structure model.

(4) Eigenvalue inversion method

The eigenvalue inversion method is mainly to solve the eigenvalue inversion of the system. The prerequisite is to know the complete spectral matrix of the system. In order to obtain a complete spectral matrix, the test value and the analysis value are generally used at the same time, and the structural spectral matrix is finally obtained through the modal expansion technology, which is also very computationally intensive. The eigenvalue inversion method is mostly used in the modification process of structural design, and rarely used in the modification of structural finite element models.

\subsection{Experimental Results}

For multi-scale reinforced concrete column images with complex background interference and multiple types 
of damage information, with different camera internal and external parameters, multi-type damage classification and identification based on regional recommendation mechanism are carried out. Regional positioning research, for accurate spatial positioning of key structural components and local damage, and correction, updating, and re-analysis of multi-scale structural models that take into account real damage information and the evolution of space-time distribution, so as to achieve the safety of the in-service structure degradation status and service performance The assessment laid the foundation.

(1) Concrete bridge deck normal forward stress

The self-weight of the structure is calculated based on the bulk weight of the material. The bulk weight of steel is $78.5 \mathrm{kN} / \mathrm{m}^{3}$, and the bulk weight of reinforced concrete is $26 \mathrm{kN} / \mathrm{m}^{3}$. According to the design and construction process, the stress state of the bridge during the construction phase is calculated.

— Top -max — Top-min — Bottom-max — Bottom-min

Concrete bridge deck normal forward stress

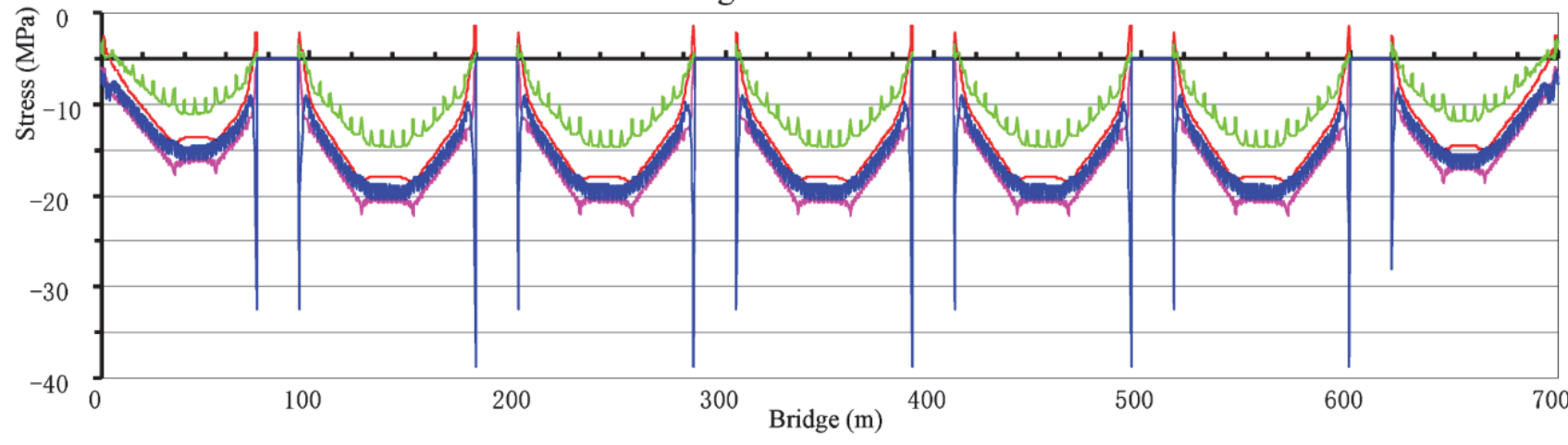

Figure 5 Damage to concrete bridge deck

Steel beam bottom plate normal bridge stress

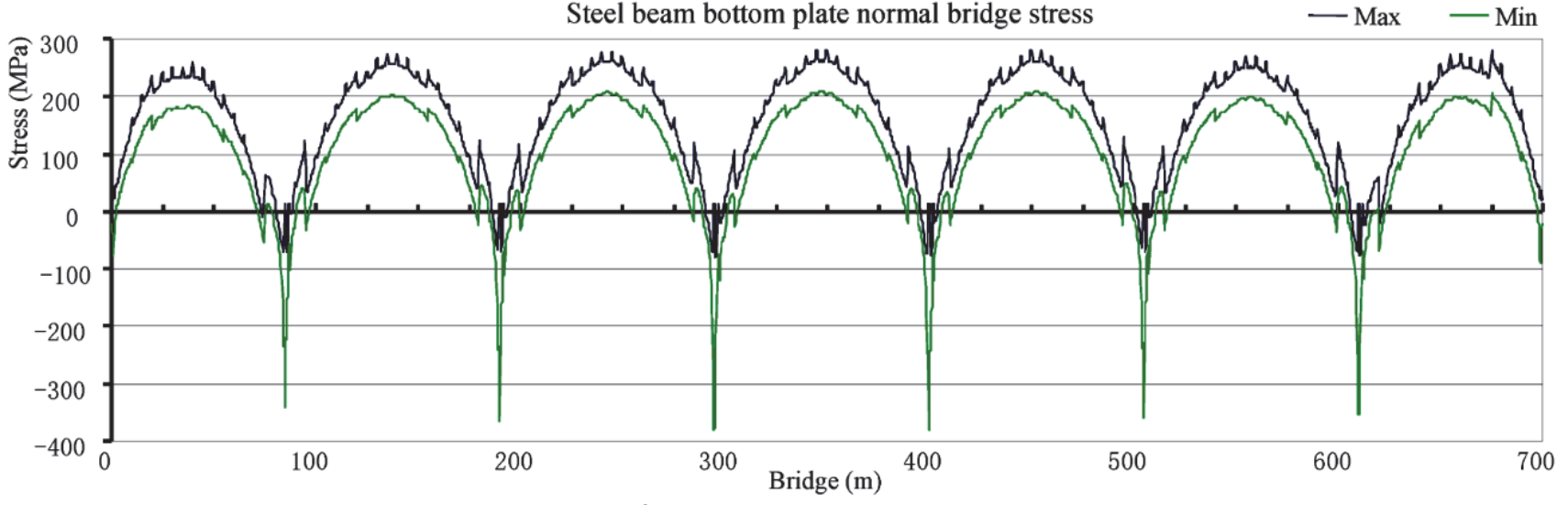

Figure 6 Steel beam bottom plate normal bridge stress

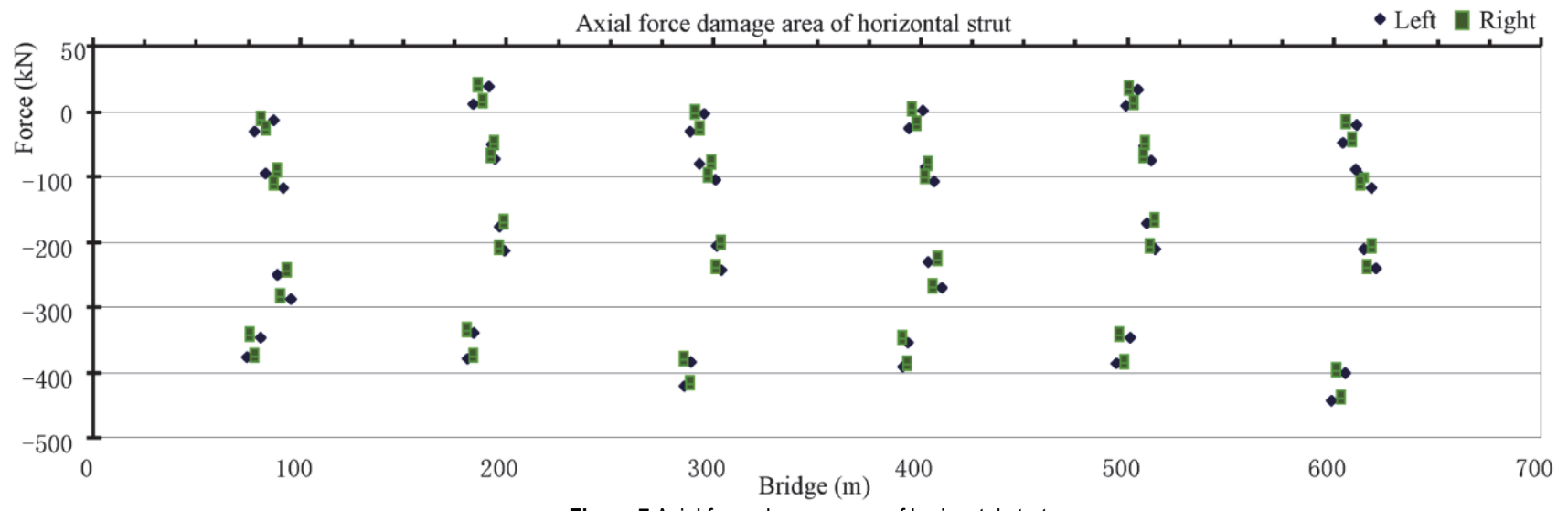

Figure 7 Axial force damage area of horizontal strut

Fig. 5 shows the distribution of normal stresses along the bridge in various stages and in the completed state of the bridge slab of the main girder. It can be seen that during the operation, especially during the fulcrum lifting stage, successive local pouring of the bridge deck slab will produce large local stresses. Hence, the bridge is most damaged here; the tensile stress and compressive stress levels will be higher.

Fig. 6 shows the normal stress distribution in the forward direction of the bridge under the load combination 1. It can be seen from the figure that the maximum tensile stress of the steel beam bottom is about $110 \mathrm{MPa}$ and the 
maximum compressive stress is about $170 \mathrm{MPa}$.

Fig. 7 shows the axial force diagram of steel beam horizontal bracing members under load combination at 600 $\mathrm{m}$. It can be seen from the figure that the maximum axial pressure of the horizontal strut member is about $270 \mathrm{kN}$. Hence, the bridge is most damaged here.

(2) Recognition effect

Fig. 8 shows the longitudinal damage distribution of the welding nails after the construction stage 2 at the end of the girders. It can be seen that the damage of the welding nail has a sudden change at the fulcrum of the pre-bending by the consideration of the pre-bending process, corresponding to the damage of the main beam, and there is a zigzag mutation at the change of the welding nail spacing. The force is near the end of the $x=700 \mathrm{~m}$ precast section, which is about $1.5 \mathrm{~mm}$.

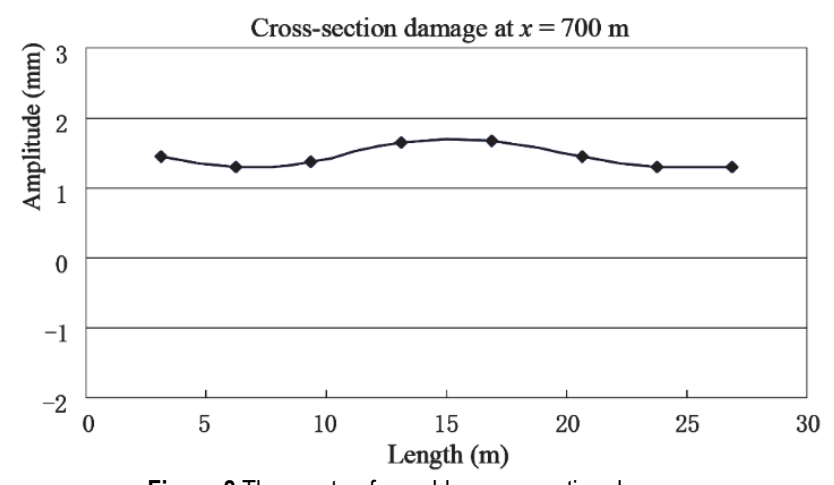

Figure 8 The most unfavorable cross-section damage

Fig. 9 is the most unfavorable cross-section damage of the welding nail at $15{ }^{\circ} \mathrm{C}$. It can be seen that after the concrete is heated up to $15{ }^{\circ} \mathrm{C}$, the maximum longitudinal damage at the end fulcrum is about $2.4 \mathrm{~mm}$, and the longitudinal damage becomes very small after the length of $1 / 10$ span, which basically does not exceed $6 \mathrm{kN}$. Moreover, the damage of the welding nail approximately the end fulcrum caused by the temperature rise of $15^{\circ} \mathrm{C}$ is the same as that caused by the vertical load, which is not good for the welding nail.

Cross-section damage at $x=700 \mathrm{~m}$ by heating up 15

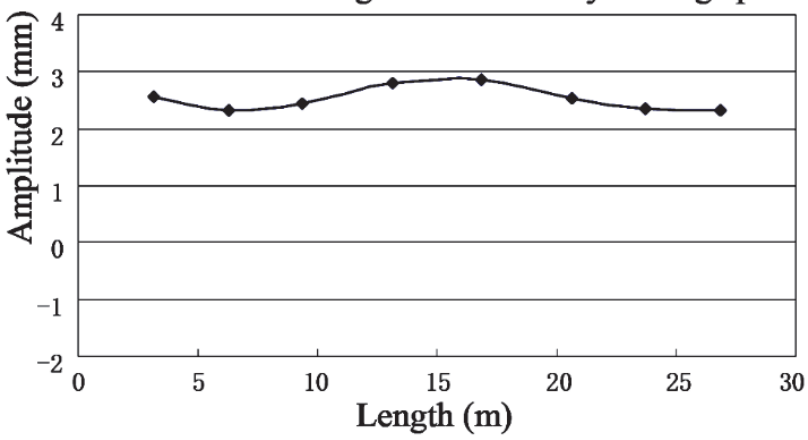

Figure 9 The most unfavorable cross-section damage by heating up $15^{\circ} \mathrm{C}$

Due to the in-depth research and application of the holistic evaluation technology based on vibration information in the aerospace, machinery and other fields, this type of technology has been used as the most important holistic evaluation method in civil structures in addition to non-destructive testing technology, and has been widely studied. The environmental vibration can obtain the vibration measurement data is that structural vibration information, so this method has the potential of real-time monitoring and has become a large important means of bridge health monitoring. On the contrary, comprehensive static response detection of long-span bridges is difficult to achieve technically and economically, and it will affect the normal operation of the bridge. The static response often does not reflect the overall working performance and damage of the bridge. It more reflects the working performance and damage of local members of the bridge.

\section{CONCLUSION}

This paper mainly summarizes the current research status of bridge safety performance evaluation systems based on health monitoring data, and the processing of massive data has always been a not well-resolved subject. This paper proposes a bridge structure damage and health diagnosis method based on health monitoring. It presents the architecture and components of bridge structural health monitoring system. The bulk weight of steel is $78.5 \mathrm{kN} / \mathrm{m}^{3}$, and the bulk weight of reinforced concrete is $26 \mathrm{kN} / \mathrm{m}^{3}$. The most unfavorable cross-section damage of the welding nail heated up to $15^{\circ} \mathrm{C}$, the maximum longitudinal damage at the end fulcrum is about $24 \mathrm{~mm}$, and the longitudinal damage becomes very small after the length of 1/10 span. As China was at the climax of transportation and largescale bridge construction at that time, the background of this era provided us with a historical opportunity. The health monitoring technology of bridge structures has developed rapidly and achieved significant results. However, due to the lack of necessary data screening, the important data flooding, or early warning systems are difficult to meet the requirements or even errors, which seriously affects the operation of use.

\section{Acknowledgement}

This paper is supported by the National Natural Science Foundation of China (51808259).

\section{REFERENCES}

[1] Wang, G., Li, F., Liu, Z., \& Meng, G. (2017). Research on ultrasonic guided wave-based damage localization for pipeline structure. Journal of Vibration Measurement \& Diagnosis, 37(3), 440-448.

[2] de Oliveira, M. A., de Souza Araujo, N. V., Inman, D. J., \& Filho, J. V. (2017). Kappa-pso-fan based method for damage identification on composite structural health monitoring. Expert Systems with Applications, 95, 1-13. https://doi.org/10.1016/j.eswa.2017.11.022

[3] Huang, J., Li, D., Li, H., Song, G., \& Liang, Y. (2018). Damage identification of a large cable-stayed bridge with novel cointegrated kalman filter method under changing environments. Structural Control \& Health Monitoring, (4), e2152. https://doi.org/10.1002/stc.2152

[4] Soman, R., Kyriakides, M., Onoufriou, T., \& Ostachowicz, W. (2017). Numerical evaluation of multi-metric data fusion based structural health monitoring of long span bridge structures. Structure \& Infrastructure Engineering, 14(6), 112. https://doi.org/10.1080/15732479.2017.1350984

[5] Kiyotaka, N., Hiroki, O., Hiroki, F., \& Yoshinori, Y. (2017). Mri-based brain healthcare quotients: a bridge between neural and behavioral analyses for keeping the brain healthy. Plos One, 12(10), e0187137. 
https://doi.org/10.1371/journal.pone.0187137

[6] Jing-xian, S. \& Jiang, F. (2018). Benchmark model correction of monitoring system based on dynamic load test of bridge. IOP Conference Series Earth and Environmental Science, 128(1), 012015. https://doi.org/10.1088/1755-1315/128/1/012015

[7] Tong, X. (2018). A preliminary research on wireless cantilever beam vibration sensor in bridge health monitoring. Frontiers of Structural and Civil Engineering, 12(2), 207214. https://doi.org/10.1007/s11709-017-0406-x

[8] Inkoom, S. \& Sobanjo, J. (2018). Availability function as bridge element's importance weight in computing overall bridge health index. Structure and Infrastructure Engineering, 14(1), 1-13. https://doi.org/10.1080/15732479.2018.1476561

[9] Poskus, E., Rodgers, G. W., Zhou, C., \& Chase, J. G. (2017). Damage identification for hysteretic structures using a mode decomposition method. Computer-aided Civil \& Infrastructure Engineering, 33(1). https://doi.org/10.1111/mice.12317

[10] Jiao, P., Borchani, W., Hasni, H., \& Lajnef, N. (2017). A new solution of measuring thermal response of prestressed concrete bridge girders for structural health monitoring. Measurement Science \& Technology, 28(8), 085005. https://doi.org/10.1088/1361-6501/aa6c8e

[11] Hasni, H., Jiao, P., Lajnef, N., \& Alavi, A. H. (2018) Damage localization and quantification in gusset plates: a battery-free sensing approach. Structural Control \& Health Monitoring, 25(2), e2158. https://doi.org/10.1002/stc.2158

[12] Rogers, M., Lemmen, K., Kramer, R., Mann, J., \& Chopra, V. (2017). Internet-delivered health interventions that work: systematic review of meta-analyses and evaluation of website availability. Journal of Medical Internet Research, 19(3), e90. https://doi.org/10.2196/jmir.7111

[13] Alavi, A. H., Hasni, H., Jiao, P., Borchani, W., \& Lajnef, N. (2017). Fatigue cracking detection in steel bridge girders through a self-powered sensing concept. Journal of Constructional Steel Research, 128, 19-38. https://doi.org/10.1016/j.jcsr.2016.08.002

\section{Contact information:}

\section{Chunguang HANG}

School of Civil Engineering,

Jiaying University, Meizhou, China

Xu LUO

(Corresponding author)

School of Mechanics and Construction Engineering,

Jinan University, Meng Minwei Science and Technology Building,

No. 601 Huangpu Avenue West, Tianhe District,

Guangzhou 510632, China

E-mail: tluoxu_jnu@163.com

\section{Xinping LI}

School of Civil Engineering and Transportation,

South China University of Technology,

Guangzhou, China

\section{Xinsha FU}

School of Civil Engineering and Transportation, South China University of Technology,

Guangzhou, China

\section{Lixiong GU}

School of Civil Engineering and Transportation, South China University of Technology,

Guangzhou, China

\section{Zhiyong OUYANG}

School of Civil Engineering,

Jiaying University, Meizhou, China 